\title{
Observational aspects of prominence oscillations
}

\author{
Oddbjørn Engvold \\ Institute of Theoretical Astrophysics \\ University of Oslo \\ PO Box 1029, Blindern \\ N-0315 Oslo, Norway \\ email: oengvold@astro.uio.no
}

\begin{abstract}
Seismology has become a powerful tool in studies of the magnetic structure of solar prominences and filaments. Reversely, analytical and numerical models are guided by available information about the spatial and thermodynamical structure of these enigmatic structures. The present invited paper reviews recent observational results on oscillations and waves as well as details about small-scale structures and dynamics of prominences and filaments.
\end{abstract}

\section{Introduction}

The oscillating nature of solar filaments (called prominences when observed beyond the limb) was first noticed as "winking filaments" (Ramsey and Smith, 1966). Subsequent studies of periodic changes in the line-of-sight velocity in prominences beyond the limb (Bashkirtsev and Mashnisch 1993; Balthasar et al. 1993; Molowny-Horas et al. 1997) and as filaments against the solar disk (Thompson and Schmieder 1991; Yi et al. 1991; Lin et al. 2003) have revealed a wide range of oscillatory periods. For an updated overviews see Ballester (2006) and Banjeree et al. (2007).

Oscillations have become a useful tool for understanding the structure and properties of the fascinating prominences and filaments (Oliver, 2001; Ballester 2006; Banjeree et al. 2007). The reason is that periods, amplitudes, wave velocities and other characteristics are inevitably closely connected with the magnetic and thermodynamic structure of the oscillating bodies. Reversely, information on relevant thermodynamic and magnetic structures and scales are essential for identification of types and modes from the observed signals.

Prosperous development of solar telescopes and instruments, ground-based and in space, combined with powerful real-time and post-processing techniques (van Noort et al. 2005), have pushed the resolution limit close to 0.2 arc sec and led to an overall enhanced quality of now available solar observations. A brief review of observational aspects of prominence oscillations follows below.

\section{Spatial and temporal characteristics of Prominences and Filaments}

\subsection{Environments}

White light eclipse images demonstrate that solar prominences are located within a dark cavity under coronal helmet-like, magnetic arches (see Saito and Hyder 1968; TandbergHanssen 1998). The helmet streamer structure represents magnetic arches that are strapping regions of photospheric opposite polarity magnetic fields. Seen against the disk solar filaments are located above and run along this so-called Polarity Inversion Line (PIL), i.e. where $\mathrm{Bz}=0$. Early soft X-ray and EUV observations (Serio et al. 1978; Schmahl 
1979) demonstrated that the cavities encompass the filaments and run along the polarity reversal boundary in filament channels.

The assumed low density cavity constitutes a region in which magnetic field orientation changes from largely along the filament channel to nearly perpendicular to the same by the overlying arcades (cf. Martin 1998). The magnetic fields of coronal cavities serve a key role in keeping the flat and non-bipolar filament field within the channels.

\subsection{Spatial Structures}

A typical solar filament is composed of a spine, barbs, and two extreme ends. The spine defines the upper horizontal part of a filament. Barbs are lateral structures that extend from some of the spine to the chromosphere. The ends, also called legs, may be a collection of threads that appear to terminate at a single point or at multiple points. These components of filaments differ in appearance and relative importance in filaments in active regions, in quiescent filaments, and in intermediate filaments. Intermediate filaments are those between active region filaments and quiescent filaments, two categories at the extreme ends of the spectrum of filaments (Lin et al. 2007).

Recent high-resolution observations by Lin (2005) and Lin et al. (2003, 2005) obtained with the Swedish 1-m Solar Telescope (SST; Scharmer et al. 2003) show fine detail everywhere within filaments (Martin et al. 2007). Such high-resolution $\mathrm{H} \alpha$ images demonstrate that filament spines, barbs and ends are all composed of thin threads. Figure 1 shows two high resolution $\mathrm{H} \alpha$ filtergrams of quiescent filaments observed with Swedish Solar Telescope, La Palma. The thread-like structures appear to be present in filaments everywhere and at all times. Martin et al. (2007) claim that the entire range of filaments that have spines and barbs, from those in active regions to those on the quiet Sun, should be amenable to theoretical description in a single model.

By inference, the ends of the filament are originating at the boundaries of positive network fields and terminating at the edges of negative network fields. Because the field direction in the barbs is consistent with the field direction in the spine, it is then implied in the model (although not yet proven from observations) that the barbs terminate in the minority magnetic polarity.
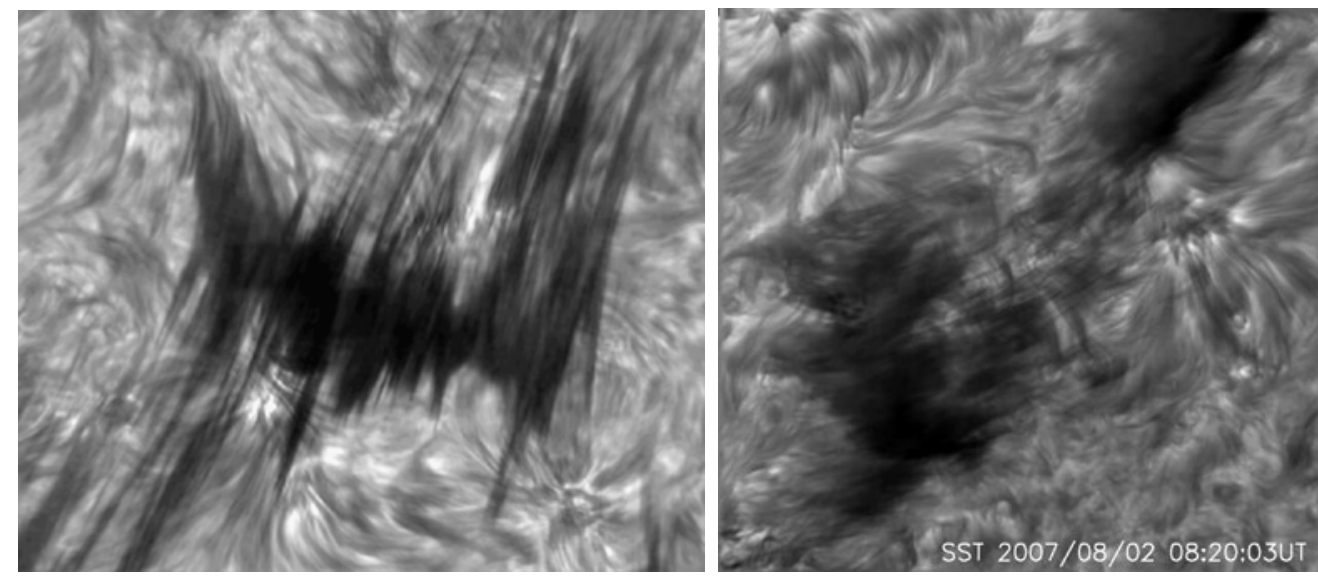

Figure 1. High resolution $\mathrm{H} \alpha$ filtergrams of two quiescent solar filaments obtained with the Swedish Solar Telescope at La Palma. Left: Close to disk center August 22, 2004. Right: SW quadrant August 2, 2007. 


\subsection{Dynamics}

Images of time series show a persistent flowing of the filament plasma along the threads in both directions with typical speeds of $10-20 \mathrm{~km} \mathrm{~s}^{-1}$. This is usually referred to as counterstreamings aligned with the magnetic field (e.g., Zirker et al. 1998; Lin et al. 2003).

Individual threads and groups of threads oscillate in the line-of-sight with periods from less than one minute to several hours (cf. Engvold, 2004). Threads are also seen to move sideways with a speed of $2-3 \mathrm{kms}^{-1}$ (e.g., Lin et al. 2005). The swaying character of these sideways motions suggests that they might be a result of Alfvén waves or kink oscillations (Engvold, Lin and Rouppe van der Voort 2008).

\subsection{Thermal structure}

The visibility, i.e. darkness, vary noticeably along the length of a thread as seen in $\mathrm{H} \alpha$ against the background chromosphere. Some observed dark threads even appear detached from the main body of the filament, as illustrated in the lower frame of Figure 1. This might be accounted for by variation in absorbing contrast along a thread that imply changes in temperature or density. Changes in contrast are thought to be a result of moderate variations in temperature and/or density in the direction of the magnetic field. The optical density of Balmer $\mathrm{H} \alpha$ is a function of the population of energy level $\mathrm{n}$ $=2$ in Hydrogen, which is proportional to electron density $\mathrm{n}_{e}^{2}$ (Engvold 1980) and to brightness temperature relative to the chromosphere below. Hence, moderate changes in temperature and density may thus render an observable thread invisible, and vice versa.

Numerous studies have shown that Hydrogen is partly ionized in solar prominences (see Heinzel et al. 1987; Gouttebroze et al. 1993).

Cirgliano et al. (2004) concluded from Differential Emission Measure from EUV spectral observations covering emission lines ranging in temperature from $\leqslant 310^{4} \mathrm{~K}$ to more than $310^{5} \mathrm{~K}$ that there is at least 40 times more emitting material at the highest temperature compared with that of plasma seen in $\mathrm{H} \alpha$. From a recent study of Ly $\alpha$ and $\operatorname{Ly} \beta$ line profiles Vial et al. (2007) conclude that prominence fine-structure is associated with strong temperature gradients perpendicular to the magnetic field.

\subsection{The overall character of oscillating threads}

Modeling of oscillations and waves in solar filaments shall have to consider the overall physical character of the threads.

The filament threads, the fundamental structure of solar filaments and prominences have widths of 0.2 and 0.3 arc sec, which also are close to the resolution limit of current best solar telescopes (cf. Lin et al. 2005). Therefore, some threads may be even thinner. The plasma parameters are similar to those of the chromosphere, which typically ranges from $\mathrm{T}_{e}=710^{3} \mathrm{~K}$ to $10^{4} \mathrm{~K}$ and $\mathrm{n}_{e}=10^{10}$ to $10^{11} \mathrm{~cm}^{-3}$ (cf. Engvold et al. 1990). One foresees rather moderate variation in the key parameters of the plasma along a thread, whereas there is clear evidence for temperature gradients in the direction perpendicular to the magnetic field (Vial et al. 2007). The fact that prominence plasma is partly ionized (Gouttebroze et al. 1993) may give rise to wave damping (Forteza et al. 2007; Solér et al. 2007a) and thereby also contribute to the flowing of the plasma (Pécseli and Engvold 2000). Last but not least, the ubiquitous plasma flows along the threads at velocities close to the acoustic speeds adds complexity to numerical modeling of filament and prominence oscillation. 


\section{Oscillations}

\subsection{Observations}

Filament and prominences oscillations have traditionally been measured from the resulting Doppler signals. With access to telescopes and instruments with gradually better spatial resolution oscillations can also be detected from motions in the plane of the sky.

Oscillations in quiescent prominences are historically classified according to their periods (cf. Solér, Oliver and Ballester, 2007a):

\begin{tabular}{lc}
\hline Type & Period \\
\hline Short & $\mathrm{P}<10 \mathrm{~min}$ \\
Intermediate & $10 \mathrm{~min}<\mathrm{P}<40 \mathrm{~min}$ \\
Long & $\mathrm{P}>40 \mathrm{~min}$ \\
\hline
\end{tabular}

Small-amplitude oscillations, $\Delta \mathrm{v}=0.1-3 \mathrm{~km} \mathrm{~s}^{-1}$, are detected at all periods, whereas large-amplitudes (20-40 $\left.\mathrm{km} \mathrm{s}^{-1}\right)$ are commonly observed at long periods.

\subsection{Large amplitude oscillations}

Large amplitude oscillations are traditionally believed to be triggered by flare associated waves (Ramsey and Smith 1966; Eto et al. 2002). However, recent studies by Jing et al. $(2003,2006)$ and Vrsnak et al. $(2007)$ have shown large amplitude waves $\left(\Delta v_{l o s}>20 \mathrm{~km}\right.$ $\mathrm{s}^{-1}$ ) propagating along the filament bodies. In these two cases the oscillations appears to be triggered by, respectively, a nearby sub-flare and by local emergence of magnetic flux. Isobe et al. (2007) observed large-amplitude oscillations during the slow rise, preeruption phase of a high latitude filament. The oscillation was observed only in part of the filament and appeared to be standing oscillation rather than a propagating wave.

\subsection{Intermediate and short amplitudes}

See Oliver and Ballester (2002) for a review. The longer period oscillations are common over the whole filament, whereas individual threads oscillate independently at shorter periods (Lin et al. 2003).

The fine threads of prominences and filaments are shown to be rooted in the photosphere below (Lin et al. 2005), where the dynamic granulation is constantly pushing them around at speeds of a few $\mathrm{km} \mathrm{s}^{-1}$. This and possible episodic interactions between constantly moving individual threads is very likely sources of the observed oscillations.

\subsection{Extremely long-period oscillations}

Foullon et al. (2004) reported on the detection of extremely long-period oscillations, with dominant period of $12.1 \mathrm{hrs}$, which were observed uninterruptedly with SoHO/EIT $195 \AA$ for nearly 6 days. In addition, the filament underwent cyclic intensity variations.

\subsection{Wave damping}

The damping time of oscillations in solar filaments and prominences range usually from 1 to 3 times the corresponding period (Molowny-Horas et al. 1997; Oliver and Ballester 2002; Lin et al. 2003; Vrsnak et al. 2007).

\subsection{Evidence of Alfvén waves}

Okamoto et al. (2007) find, from new observations with Hinode, fine-scale threadlike prominence structures oscillating in the plane of the sky with periods of $130-250$ s and amplitudes ranging between 200 and $850 \mathrm{~km}$. The oscillations were coherent over a length of $16000 \mathrm{~km}$. The authors conclude that the oscillations is a signature of Alfvén waves 
propagating along magnetic fields of the prominence. Engvold et al (2008) observe swaying motions of thin filament threads of amplitudes $\sim 90 \mathrm{~km}, 3-4 \mathrm{~min}$ periods and phase speeds $>100 \mathrm{~km} \mathrm{~s}^{-1}$ suggesting the presence the presence of Alfvén waves. Jensen et al. (1994) found that low amplitude oscillations in filaments very likely are associated with Alfvén waves. Régnier et al. (2001) arrived at a similar conclusion from their study of oscillations of an active region filament within which they identified the observed frequencies in terms of Alfvén waves. On the other hand, given the very fine-scale, thread-like structured magnetic field in solar filaments Erdélyi and Fedun (2007) point out that Alfvénic perturbations would rather give rise to torsional oscillations, and that the nature of the reported signatures ought to be investigated further.

\section{Concluding remarks}

The complicated pattern of oscillations in highly structured solar filaments and prominences is a challenge to observers as well as to theoreticians and modelers. Analytical and numerical models of the oscillations are guided by available information on the intricate, dynamic nature of these enigmatic structures.

The current picture of solar prominences and filaments which may serve as a guide in ongoing modeling of oscillations, are summarized as follows:

- The unique magnetic configuration of filaments and prominences is under the influence and control by the magnetic fields of the surrounding filament channels, cavities and the overlying coronal arches constituting the upper boundaries of the channels.

- All types of filaments and prominences consist of magnetic threads of thickness $\leqslant 0.3$ arc sec. The true thread lengths are very likely ranging from a typical supergranule diameter to the full length of the filament.

- Usually one sees only parts of the full length of a thread in $\mathrm{H} \alpha$ and other chromospherictype lines. Rather moderate variations in plasma density and/or temperature along the thread may account for the noted spatial and temporal changes in visibility.

- There appears to be a weak coupling between the threads that allows them to oscillate both individually (shorter periods) and together (longer periods).

- It is generally assumed, but not yet proven, that the cool threads are imbedded in thin, hot coronal plasma; this implies that each thread has a PCTR.

- Plasma is flowing along all threads all of the time, and in opposite directions in neighboring threads, at speed up to $20 \mathrm{~km} \mathrm{~s}^{-1}$ in quiescent filaments. Higher flow speed are observed in AR filaments.

\section{Acknowledgement}

The author thanks Yong Lin, Sara F. Martin and Luc Rouppe van der Voort for inspiring and helpful discussions as well as for various input to this review.

\section{References}

Ambroz, P. and Schroll, A. 2000, J. Astrophys. Astron., 21, 205

Ballester, J.L. 2006, Space Science Review, 122, 129

Banjeree, D., Erdélyi, R., Oliver, R., and O'Shea, E. 2007, Solar Phys., 246, 3

Bashkirtsev, V.S. and Mashnich, G.P. 1993, Astron. Astrophys., 279, 610

Cirgliano, K., Vial, J.-C., and Rovira, M. 2004, Solar Phys., 223, 94

Diaz, A.J., Oliver, R., and Ballester, J.L. 2005, Astro. Astrophys., 440, 1167

Diaz, A.J. and Roberts, B. 2006, Solar Phys., 236, 111

Engvold, O. 1980, Solar Phys., 67, 351 
Engvold, O., Hirayama, T., Leroy, J.L., Priest, E.R. and Tandberg-Hanssen, E. 1990, Proceedings of the IAU Symposium 117, "Dynamics of Quiescent Prominences". Eds: V. Rudjak and E. Tandberg-Hanssen, p. 294

Engvold, O. 2004, Proceedings of the IAU Symposium, 223, "Multi-Wavelength Investigations of Solar Activity". Editors: A.V. Stepanov, E.E. Benevolenskaya, and A.G. Kosovichev, p. 187

Engvold, O., Lin, Y. and Rouppe van der Voort, L. 2008, Solar Phys., in preparation

Erdélyi, R. and Fedun, V. 2007, Science, 318, 1572

Eto, S., Isobe, H., Narukage, N., Asai, A., Morimoto, T., Thompson, B., Yashiro, S., Wang, T., Kitai, R., Kurokawa, H., and Shibata, K. 2002, Publ. Astron. Soc. Japan, 54, 481

Forteza, P., Oliver, R., Ballester, J.L. and Khodachenko, M.L. 2007, Astron. Astrophys., 461, 731

Foullon, C., Verwichte, E., and Nakariakov, V. 2004, Astron. Astrophys., 427, L5

Gouttebroze, P., Heinzel, P., and Vial, J.-P. 1993, Astron. Astrophys. Suppl. Ser, 99, 513

Heinzel, P., Gouttebroze, P. and Vial, J.-P. 1987, Astron. Astrophys., 183, 351

Isobe, H., Tripathi, D., Asai, A. and Jain, R. 2007, Solar Phys., 246, 89

Jensen, E., Yi, Z. and Engvold, O. 1994, Solar Phys., 149, 209

Jing Ju, Lee Jeongwoo, Spirock, T.J., Xu Yan, and Wang Haimin. 2003, Astrophys. J., 584, L103

Jing Ju, Lee Jeongwoo, Spirock, T.J., and Wang Haimin. 2006, Solar Phys., 236, 97

Lin, Y. 2005, PhD Thesis, University of Oslo

Lin, Y., Wiik, J.E. and Engvold, O. 2003, Solar Phys., 216, 109

Lin, Y., Engvold, O., Rouppe van der Voort, L., Wiik, J.E., and Berger, T.E. 2005, Solar Phys., 226,239

Martin, S.F. 1998, Solar Phys. 182, 107

Martin, S.F., Lin, Y., and Engvold, O. 2007, Solar Phys. (in press)

Molowny-Horas, R., Oliver, R., Ballester, J.L. and Baudin, F. 1997, Solar Phys., 172, 181

Okamoto, T.J., Nakai, H., Keiyama, A., Narukage, N., UeNo, S., Kitai, R., Kurokawa, H. and Shibata, K. 2004, Astrophys. J., 608, 1124

Okamoto, T.J., Tsuneta, S., Berger, T.E., Ichimoto, K., Katsukawa, Y., Lites, B.W., Nagata, S., Shibata, K., Shimitzu, T., Shine, R.A., Suematsu, Y., Tarbell, T.D. and Title, A.M. 2007, Science, 318, 1577

Oliver, R. 2001, Encyclopedia of Astronomy and Astrophysics, Institute of Physics Publishing, Bristol and Philadelphia, Ed.: P. Murdin 3, 2707

Oliver, R., Ballester, J.L. 2002, Solar Phys., 206, 45

Pécseli, H. and Engvold, O. 2000, Solar Phys., 194, 73

Ramsey, H.E. and Smith, S.F. 1966, Astron. J., 71, 197

Régnier, S., Solomon, J. and Vial, J.-C. 2001, Astron. Astrophys., 376, 292

Saito, K. and Hyder, C. 2007, Solar Phys., 5, 61

Scharmer, G.B., Bjelksjo, K., Korhonen, T.K., Lindberg, B., and Petterson, B. 2003, Proceedings of the SPIE, Volume 4853, "Innovative Telescopes and Instrumentation for Solar Astrophysics" Editors: S.L. Keil \& S.V. Avakyan, p. 341

Schmahl, E.J. 1978, in IAU Colloquium 44 - The Physics of Solar Prominences, Eds. E. Jensen, P. Maltby and F. Orrall, 102

Serio, S. Vaiana, G.S., Godoli, G. Motta, S., Pironello, V. and Zappala, R.A. 1978, Solar Phys., 59,65

Solér, R., Oliver, R. and Ballester, J.L. 2007a, Astron. Astrophys., 471, 1023

Solér, R., Oliver, R. and Ballester, J.L. 2007b, Solar Phys., 246, 73

Tandberg-Hanssen, E. 1995, The Nature of Solar Prominences, Kluwer Academic Publisher

Thompson, W.T. and Schmieder, B. 1991, Astron. Astrophys., 243, 501

van Noort, M., Rouppe van der Voort, L., and Löfdahl, M. 2005, Solar Phys., 228, 191

Vial, J.-C., Ebadi, H, and Ajabshirizadeh, A. 2007, Solar Phys., 246, 327

Vrsnak, B., Veronig, A.M., Thalmann, J.K., and Zic, T. 2007, Astron. Astrophys., 471, 295

Zirker, J.B., Engvold, O. and Martin, S.F. 1998, Nature, 396, 440 